\title{
Status and perspectives of the Dubna superheavy element factory
}

\author{
Sergey Dmitrieva ${ }^{\mathrm{a}}$, Mikhail Itkis, and Yuri Oganessian \\ Flerov Laboratory of Nuclear Reactions, Joint Institute for Nuclear Research, 141980 Dubna, \\ Russian Federation
}

\begin{abstract}
In the Flerov Laboratory of Nuclear Reactions, Joint Institute for Nuclear Research (FLNR JINR), construction of a new experimental complex is currently in progress (Superheavy Element Factory), aimed at the synthesis of new superheavy nuclides and the detailed study of those already synthesized. The project includes the construction of a new accelerator of stable and long-lived isotopes in the mass range $A=10-100$ with an intensity of up to $10 \mathrm{p} \mu \mathrm{A}$ and energy up to $8 \mathrm{MeV} /$ nucleon; construction of a new experimental building and infrastructure for housing the accelerator with five channels for the transportation of beams to a $1200-\mathrm{m}^{2}$ experimental hall that is equipped with systems of shielding and control for operations with radioactive materials; development of new separators of reaction products; upgrade of the existing separators and development of the new detection modules for the study of nuclear, atomic, and chemical properties of new elements. The first experiments are planned for 2018.
\end{abstract}

\section{Introduction}

The six heaviest chemical elements with atomic numbers 113 to 118 that fill the $7^{\text {th }}$ row of Mendeleev's Periodic Table were synthesized in reactions of ${ }^{48} \mathrm{Ca}$ ions with actinide targets in the experimental studies carried out over the recent years. Over 50 new isotopes of elements 104 to 118 with maximum neutron excess were for the first time produced and their decay properties were determined in these investigations [1, 2]. The new isotopes considerably filled up the Chart of the Nuclides and expanded it up to $Z=118$ and $N=177$ superheavy elements. Fundamental conclusions of the modern theory concerning the limits of existence of nuclear matter have for the first time received experimental confirmation.

The discovery of the new domain (island) of stability and the very fact of existence of superheavy elements (SHE) have posed a number of new questions associated with fundamental properties of nuclear matter:

- Can even heavier nuclei exist? Is the "Island of Stability of SHE" the last one on the Chart of the Nuclides?

- Can the superheavy nuclei be formed in the process of nucleosynthesis like those stable and long-lived nuclei in the groups of $\mathrm{Pt}, \mathrm{Pb}$, and $\mathrm{U}-\mathrm{Th}$ found in Nature?

- What is the electron structure of a superheavy atom in strong Coulomb field?

- What is the limit of Mendeleev's Table? How much are the chemical properties of superheavy elements similar to those of their lighter homologues?

\footnotetext{
${ }^{\text {a }}$ Corresponding author: dmitriev@jinr.ru
} 
In search for answers to these and many related questions we have to considerably widen our knowledge about the subject of investigation. Obviously, to reach this aim we need to greatly broaden the field of work and involve new facilities. However, judging by first attempts, going beyond the area of already synthesized superheavy elements is quite a difficult task.

\section{SHE factory}

Direct synthesis of elements with $Z>118$ in fusion reactions means using projectiles heavier than $\mathrm{Ca}$, since the capability of high-flux reactors to produce target material is limited to $\mathrm{Cf}$ isotopes. A number of attempts undertaken in various laboratories and aimed at the synthesis of elements 119 and 120 did not yield any result. Limits of production cross sections of nuclei with $Z=120 \mathrm{in}$, for example, the reaction ${ }^{54} \mathrm{Cr}+{ }^{248} \mathrm{Cm}$ [3] and nuclei with $Z=119$ via ${ }^{50} \mathrm{Ti}+{ }^{249} \mathrm{Bk}$ [4] were ten to twenty times lower than those of production of isotopes of elements 114 and 115 in experiments with ${ }^{48} \mathrm{Ca}$.

Advance in the other direction - towards the desired area of more neutron-rich isotopes of the known SHE closer to the predicted shell closure at $N=184$ - is possible only with use of radioactive projectiles that are more neutron-rich than ${ }^{48} \mathrm{Ca}$. Unfortunately, the intensities of beams of radioactive nuclei at the most advanced accelerators and even those in the design stage are extremely low for performing this kind of experiments.

Moving in the opposite direction - towards neutron deficit in superheavy nuclei - leads to strong decrease of production cross section when going off the shell $N=184$.

Under these circumstances, it seems appropriate to investigate in detail the already synthesized nuclei: to determine their masses, forms, and fission barriers, find their excited states, etc. Yet here we need to considerably improve the statistics. Individual events of formation and decay of superheavy nuclides that are registered in present experiments with a rate of one per day to one per month (this is considered a great achievement of the last years) are far from being sufficient for these purposes. Experimental results should be presented not by single events but by hundreds and thousands. Only then one will see rare decay channels of the superheavy nuclei or study in detail nuclear transitions to various states, which means to make complete measurements in spite of extremely low cross sections of producing superheavy elements.

Note that the present situation in some aspects reproduces the beginning of work on synthesis of superheavy elements twenty years ago when a new approach to the synthesis of SHE (reactions ${ }^{48} \mathrm{Ca}+$ actinides) was found and a significant breakthrough in experimental technique was made.

We came to the conclusion that at present, based on accumulated knowledge in study of

- reactions of synthesis of new elements (fusion reactions with evaporation of neutrons);

- cross sections of production of the nuclei of SHE reaction channels at various beam energies (excitation functions);

- competing reaction channels (background conditions);

- decay characteristics of the isotopes of SHE (decay modes, energies, decay times, etc.), as well as the achievements of the last twenty years in the fields of

- technique of physical experiment;

- physics and technology of charged particle accelerators and plasma physics;

- new highly sensitive precision detectors for nuclear radiation;

- production of new target materials in high-flux nuclear reactors and development of new target technologies, etc. 


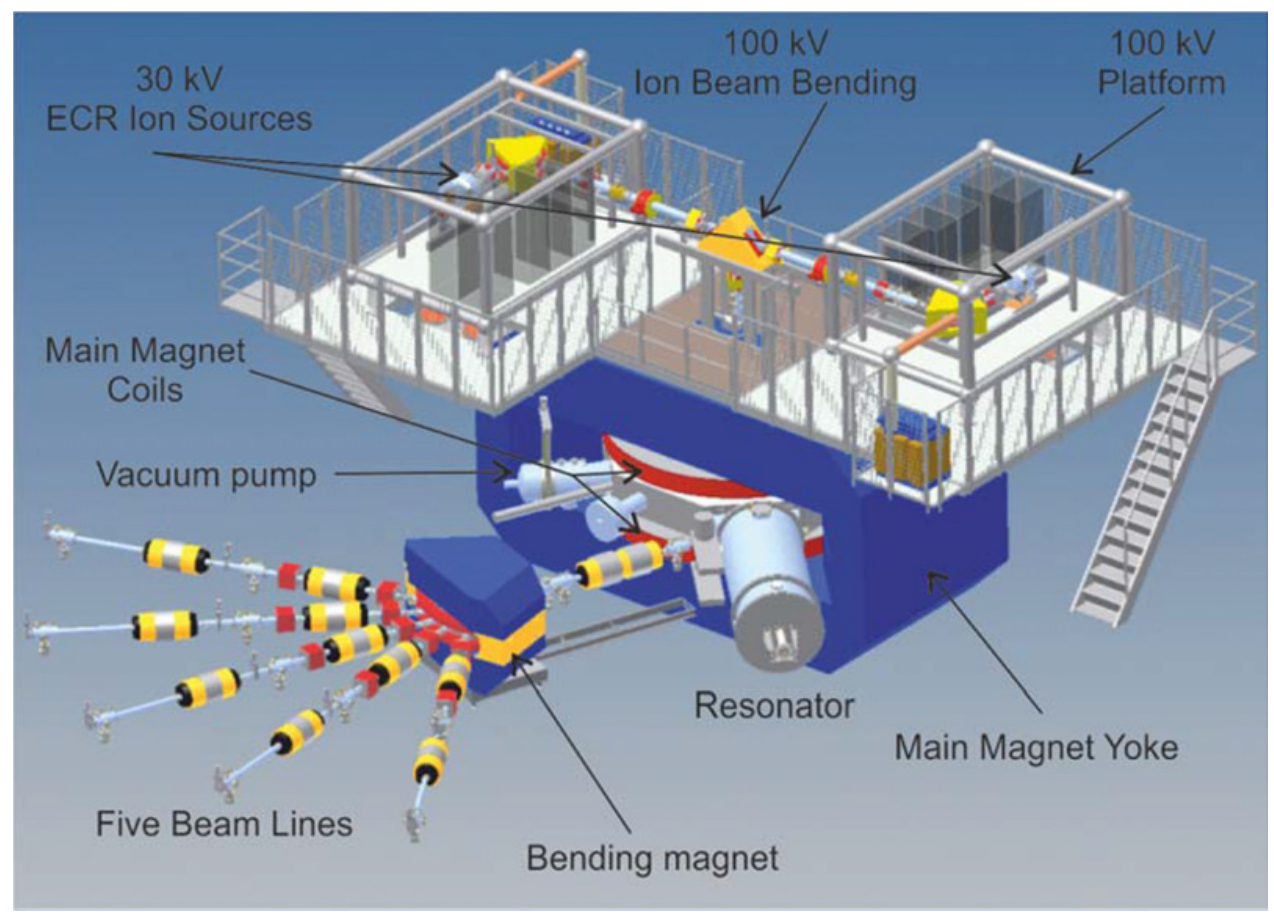

Figure 1. DC-280 cyclotron view.

Table 1. Expected parameters of the DC280 cyclotron.

\begin{tabular}{|c|c|c|}
\hline \multicolumn{3}{|c|}{ DC280 (expected parameters) } \\
\hline Ion & $\begin{array}{c}\text { Ion energy } \\
{[\mathrm{MeV} / A]}\end{array}$ & $\begin{array}{c}\text { Output Intensity } \\
{[\mathrm{pps}]}\end{array}$ \\
\hline${ }^{7} \mathrm{Li}$ & 4 & $1 \times 10^{14}$ \\
\hline${ }^{18} \mathrm{O}$ & 8 & $1 \times 10^{14}$ \\
\hline${ }^{40} \mathrm{Ar}$ & 5 & $6 \times 10^{13}$ \\
\hline${ }^{48} \mathrm{Ca}$ & 5 & $6 \times 10^{13}-1.2 \times 10^{14}$ \\
\hline${ }^{54} \mathrm{Cr}$ & 5 & $2 \times 10^{13}$ \\
\hline${ }^{58} \mathrm{Fe}$ & 5 & $1 \times 10^{13}$ \\
\hline${ }^{13} \mathrm{Xe}$ & 5 & $1 \times 10^{14}$ \\
\hline${ }^{238} \mathrm{U}$ & 7 & $5 \times 10^{10}$ \\
\hline
\end{tabular}

it is possible to increase the production rate of the known isotopes of SHE by one to two orders of magnitude and carry out experiments aimed at the synthesis of new elements with $Z>118$ by employing a new experimental complex, the "SHE Factory", and thus make a second breakthrough in the world of the heaviest nuclei and elements.

The ideas incorporated in the project of the SHE Factory are associated with developing the experimental basis in several directions. These directions are:

- construction of new powerful accelerator of stable and long-living isotopes in mass range $A=10-100$ with intensity up to $10 \mathrm{p} \mu \mathrm{A}$ and energy up to $8 \mathrm{MeV} /$ nucleon (see Fig. 1 and Table 1 for more details);

- construction of a new experimental building and infrastructure for placing the accelerator with five channels for transportation of beams to a $1200-\mathrm{m}^{2}$ experimental 

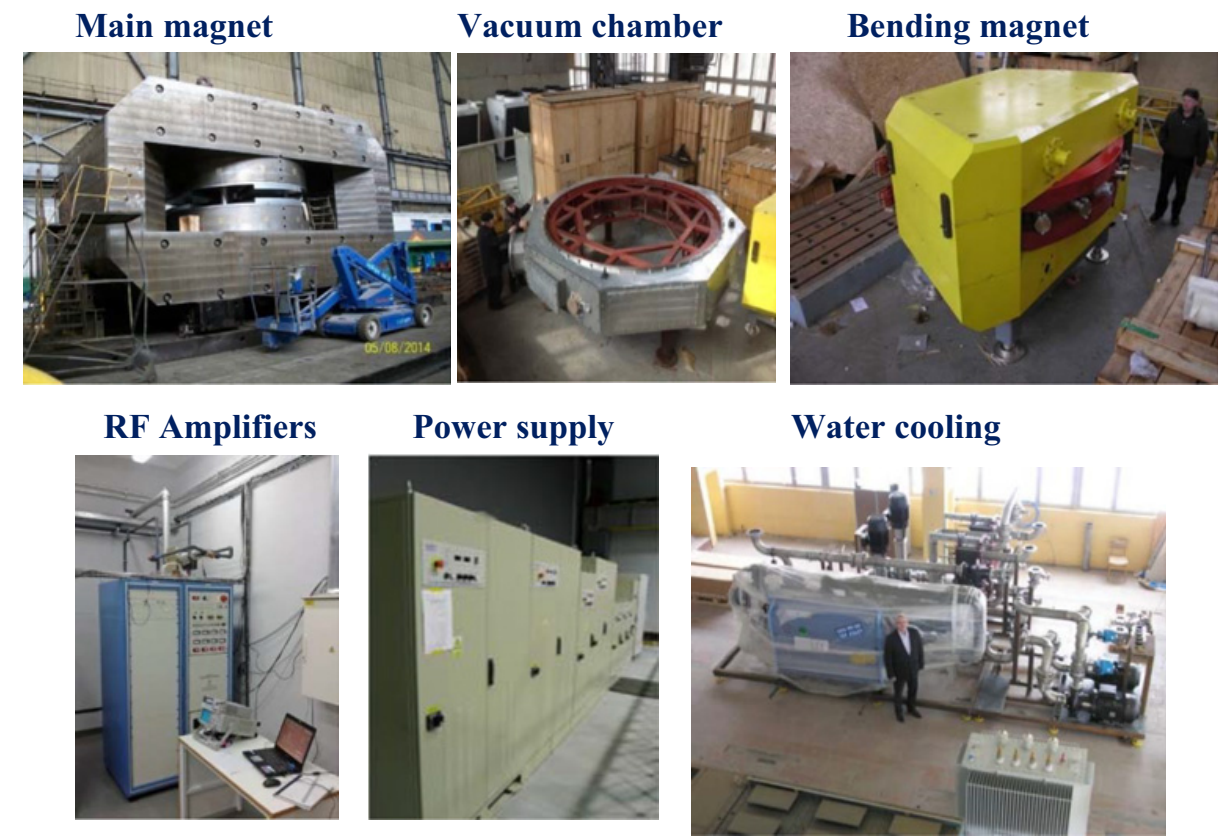

Figure 2. The main parts of cyclotron DC-280.

hall, equipped with systems of shielding and control matching the class two of operations with radioactive materials;

- development of new separating channels and development of new detection modules (including use of the already existing ones) for the study of nuclear, atomic, and chemical properties of new elements;

- production of new target materials and development of techniques of making targets with high thermal and radiation stability (running and projected nuclear reactors and enrichment facilities);

- development of a base for research with intense ion beam in related fields of science and technology.

All major parts of new cyclotron DC-280 are produced and delivered to Dubna (Fig. 2).

In general, studies of heavy and superheavy nuclei are related to atomic physics, nuclear physics, astrophysics, and radiochemistry. They cover a wide range of phenomena, the study of which is far more than experiments that are to be carried out at the SHE Factory. However, when experiments are performed with extremely heavy nuclei at the limit of stability and thus require extremely high sensitivity, advantages of the SHE Factory become highly important. This is the specificity of the new complex. Its program of experimental research will be directly connected with the achievements of world science and is designed for broad international cooperation.

A long-term program of research of SHE should be developed with regard to the development of the new complex, its phased commissioning, production of the primary beam, and start of the first experiment in 2018. Experimental research at the existing complex ECR$4 \mathrm{M}+\mathrm{U}-400+\mathrm{DGFRS}$ and at the functioning setups will continue at the same pace until the first full-scale experiment at SHE Factory (2017-2018). However, priority is given to the studies that will be continued at the SHE Factory. 
The program already begun in 2014. It can be nominally divided, with some overlap, in three phases:

- construction of the SHE Factory, design and construction of new experimental setups, research work with beams of ${ }^{48} \mathrm{Ca}$ and ${ }^{50} \mathrm{Ti}$ ions with aim of obtaining additional data for the experimental program at the SHE Factory (2014-2017);

- putting into operation of the new accelerator complex, production of the primary beam at target position, preparation of detector modules and experimental equipment for running the first experiment;

- setting up the first experiment;

- producing results that demonstrate the capabilities of the SHE Factory;

- reaching of design parameters (2018).

During the first phases (2014-2018) the scientific and technical studies are aimed at getting answers to key questions that determine further research at SHE Factory. This research includes the following:

- Study of the nuclei at the border of the "Island of Stability" of SHE. Synthesis of neutron-deficient isotopes of $\mathrm{Fl}(Z=114)$ in reactions ${ }^{48} \mathrm{Ca}+{ }^{239,240} \mathrm{Pu}$ and of new isotopes of element 118 in reactions ${ }^{48} \mathrm{Ca}+{ }^{249-251} \mathrm{Cf}$. The first experiment was carried out in 2015 [5].

- Change from ${ }^{48} \mathrm{Ca}$ to a beam of ${ }^{50} \mathrm{Ti}$ with aim of preparing experiments on the synthesis of elements 119 and 120. Model experiment on the synthesis of the isotope ${ }^{285} \mathrm{Fl}$ in reactions ${ }^{48} \mathrm{Ca}+{ }^{240} \mathrm{Pu}$ and ${ }^{50} \mathrm{Ti}+{ }^{238} \mathrm{U}$. The ${ }^{50} \mathrm{Ti}$ ion beam was obtained with the ECR+U-400 complex by using MIVOC method [6].

- Experimental investigation of characteristics of spontaneous fission of the neutron-rich nuclei with $Z=104$ to 112 obtained in decay chains of superheavy elements.

- Preparation of experiments at SHE Factory aiming on study of chemical behaviour of elements 112 to 118 compared to their light homologues. Running control experiments with a beam of ${ }^{48} \mathrm{Ca}$. Development of separator and instrumentation.

- Choice of the modes of operation of the cyclotron DC-280 and ECR source for producing intense beam of ${ }^{48} \mathrm{Ca}$ ions and carrying out the first experiment at the SHE Factory.

- Choice of design and calculation of characteristics of the separator of recoil nuclei for the first experiment with high-intensity ${ }^{48} \mathrm{Ca}$ beam at the SHE Factory. New gas-filled recoil separator has been already designed and contracted with the SigmaPhi company in France. Its commissioning is planned for the beginning of 2018.

- Alpha spectroscopy of the deformed nuclei ${ }^{257} \mathrm{Rf}$ and ${ }^{258} \mathrm{Db}$ using high-resolution detector of $\alpha$ particles in cold fusion reactions ${ }^{50} \mathrm{Ti}+{ }^{208} \mathrm{~Pb},{ }^{209} \mathrm{Bi}$ in view of carrying out experiments on spectroscopy of superheavy nuclei ${ }^{287} \mathrm{Fl}$ and ${ }^{288} 115$ and their decay products.

\section{Summary}

Currently, the Superheavy Elements Factory project is being implemented at the FLNR. It is one of the key projects in the programme of development of JINR. The launch of the Factory is scheduled for 2017/2018. The DC-280 cyclotron commissioning is planned for December 2017. First experiments with intense beams of ${ }^{48} \mathrm{Ca}$ with the use of new gas-filled separator should be carried out in the mid of 2018. 
The SHE Factory has no analogue in the world practice. It is developed with a perspective of 20 to 25 years (as all the heavy-ion accelerators at JINR) and should be in demand as an experimental complex that provides leadership in study of heavy and superheavy elements.

The reported study was partially supported by the special grant of the Moscow region government (RF).

\section{References}

[1] Yu.Ts. Oganessian and V.K. Utyonkov, Rep. Prog. Phys. 78, 036301 (2015).

[2] Yu.Ts. Oganessian et al., Phys. Rev. C 87, 014302 (2013).

[3] S. Hofmann et al., Eur. Phys. J. A52, 180 (2016).

[4] J. Khuyagbaatar et al., Phys. Rev. Lett. 112, 172501 (2014).

[5] V.K. Utyonkov et al., Phys. Rev. C92, 034609 (2015).

[6] A.V.Yeremin et al., Phys. Part. Nucl. Lett. 12, 43 (2015). 